\title{
Some Results for the Drazin Inverses of the Sum of Two Matrices and Some Block Matrices
}

\author{
Abdul Shakoor, Hu Yang, and Ilyas Ali \\ College of Mathematics and Statistics, Chongqing University, Chongqing 401331, China \\ Correspondence should be addressed to Abdul Shakoor; ashakoor313@gmail.com
}

Received 12 August 2013; Accepted 26 November 2013

Academic Editor: Kazutake Komori

Copyright (C) 2013 Abdul Shakoor et al. This is an open access article distributed under the Creative Commons Attribution License, which permits unrestricted use, distribution, and reproduction in any medium, provided the original work is properly cited.

We give a formula of $(P+Q)^{D}$ under the conditions $P^{2} Q+Q P Q=0, P^{3} Q=0$, and $P Q P Q=0$. Then, we apply it to give some expressions for the Drazin inverse of block matrix $M=\left(\begin{array}{cc}A & B \\ C\end{array}\right)$ ( $A$ and $D$ are square matrices) under some conditions, generalizing some recent results in the literature. Finally, numerical examples are given to illustrate our results.

\section{Introduction}

Let $\mathbb{C}^{m \times n}$ denote the set of $m \times n$ complex matrices. The Drazin inverse of $A \in \mathbb{C}^{n \times n}$ is the unique matrix $A^{D}$, satisfying the following equation:

$$
A^{k+1} A^{D}=A^{k}, \quad A^{D} A A^{D}=X, \quad A A^{D}=A^{D} A,
$$

where $k=\operatorname{ind}(A)$ is the index of $A$, the smallest nonnegative integer for which $\operatorname{rank}\left(A^{k+1}\right)=\operatorname{rank}\left(A^{k}\right)$ (see [1]). In particular, when $\operatorname{ind}(A)=1$, the Drazin inverse of $A$ is called the group inverse of $A$. If $A$ is nonsingular, it is clear that $\operatorname{ind}(A)=0$ and $A^{D}=A^{-1}$. Throughout this paper, we denote by $A^{\pi}=I-A A^{D}$ and define $A^{0}=I$, where $I$ is the identity matrix with proper sizes.

The importance of the Drazin inverse and its applications to singular differential equations and difference equations, Morkov chains and iterative methods, cryptography, numerical analysis, to structured matrices, and perturbation bounds for the relative eigenvalue problems can be found in [2-5].

In 1958, Drazin [6] gave a result of $(P+Q)^{D}$, where $P$ and $Q$ are square matrices, and proved that

$$
(P+Q)^{D}=P^{D}+Q^{D} \text { when } P Q=Q P=0 .
$$

In 2001, Hartwig et al. [7] derived a result of $(P+Q)^{D}$ when $P Q=0$. In 2005, Castro-González [8] provided the representation of $(P+Q)^{D}$ when $P^{D} Q=0, P Q^{D}=0$, and $Q^{\pi} P Q P^{\pi}=0$. In 2008, Castro-González et al. [9] determined the result of $(P+Q)^{D}$ when $P^{2} Q=0$ and $P Q^{2}=0$. In 2009, Martínez-Serrano and Castro-González [10] derived a formula of $(P+Q)^{D}$ when $P^{2} Q=0$ and $Q^{2}=0$. In 2011, Yang and Liu [11] established some expressions of $(P+Q)^{D}$ when $P Q^{2}=0$ and $P Q P=0$, and in 2012, Bu et al. [12] got the explicit representation of $(P+Q)^{D}$ when $P^{2} Q=0, Q^{2} P=0$, $P^{3} \mathrm{Q}=0$, and $\mathrm{QPQ}=0, Q P^{2} \mathrm{Q}=0$, respectively. Other related results have been studied in [4, 13-19].

On the other hand, a related topic is to discuss a representation of the Drazin inverse of block matrix $M=\left(\begin{array}{ll}A & B \\ C & D\end{array}\right) \epsilon$ $\mathbb{C}^{n \times n}$, where $A$ and $D$ are square matrices. Campbell and Meyer Jr. [2] first proposed an open problem to find an explicit formula of the Drazin inverse of block matrix $M=$ $\left(\begin{array}{ll}A & B \\ C & D\end{array}\right) \in \mathbb{C}^{n \times n}$, (where $A$ and $D$ are square matrices), in terms of $A, B, C$, and $D$. To find the Drazin inverse of $(P+Q)$ and $M$ in terms of $P, Q, P^{D}$, and $Q^{D}$ and $A, B, C$, and $D$, respectively, without side conditions is quite complicated and it has not been solved till now. However, many papers have been studied some special cases of this open problem and gave the representations for the Drazin inverse of $(P+Q)$ and $M$ under some conditions. Here, we list some cases of Drazin inverse of block matrix $M$ :

(i) $B C=0, D C=0$ (or $B D=0)$ and $D$ is nilpotent (see [5]);

(ii) $B C A=0, B D=0$, and $D C=0$ (or $B C$ is nilpotent) (see [9]);

(iii) $B C A=0, B C B=0, D C A=0$, and $D C B=0$ (see [11]); 
(iv) $B C=0, B D=0$, and $D C=0$ (see [20]);

(v) $B C=0$ and $D C=0$ (see [21]);

(vi) $B C A=0, B C B=0, A B D=0$, and $C B D=0$ (see $[22])$.

The generalized Schur complement of $A$ in $M$, which is stated as $S=D-C A^{D} B$, is very important to find the Drazin inverse of $M$, when the generalized Schur complement is either zero or nonsingular. Martínez-Serrano and CastroGonzález [10] gave results under the conditions $A^{2} A^{\pi} B=0$, $C A A^{\pi} B=0$, and $B C A^{\pi} B=0$ and generalized that Schur complement is equal to zero. Also, they derived the expressions of $M^{D}$ under the assumptions $A^{2} A^{\pi} B=0, C A A^{\pi} B=$ 0 , and $C A^{\pi} B=0$ and generalized Schur complement is nonsingular. The Drazin inverse of $M$ has been studied in [10, 23], when generalized Schur complement is equal to zero and also has been studied in $[5,10,24,25]$, when the generalized Schur complement is nonsingular. Some representations for the Drazin inverse of $M$ when the generalized Schur complement is nonsingular, including generalizations of the above mentioned results, will be derived in Section 4 under some conditions.

This paper is organized as follows. In Section 2, some helpful lemmas will be given. In Section 3, we give the explicit formula of $(P+Q)^{D}$ under the conditions $P^{3} Q=0, P^{2} Q+$ $Q P Q=0$, and $P Q P Q=0$ and also give a numerical example to demonstrate our result. In Section 4, we use our result to find the Drazin inverse of block matrix $M=\left(\begin{array}{ll}A & B \\ C & D\end{array}\right)$ and also to find the expression for $M^{D}$ when the generalized Schur complement is nonsingular, which can be regarded as the generalizations of some results given in $[5,20]$. Finally, in Section 5, we give two numerical examples to illustrate our results of block matrices.

\section{Some Lemmas}

In order to prove the main results, first we need the following lemmas.

Lemma 1 (see [1]). Let $A \in \mathbb{C}^{m \times n}$, and let $B \in \mathbb{C}^{n \times m}$. Then, $(A B)^{D}=A\left((B A)^{2}\right)^{D} B$.

Lemma 2 (see [7]). Let $P, Q \in \mathbb{C}^{n \times n}$. If $P Q=0$, then

$$
(P+Q)^{D}=Q^{\pi} \sum_{i=0}^{t-1} Q^{i}\left(P^{D}\right)^{i+1}+\sum_{i=0}^{t-1}\left(Q^{D}\right)^{i+1} P^{i} P^{\pi}
$$

where $t=\max \{\operatorname{ind}(P)$, ind $(Q)\}$.

Lemma 3 (see [26]). Let $M_{1}=\left(\begin{array}{ll}A & 0 \\ C & B\end{array}\right)$, and let $M_{2}=\left(\begin{array}{ll}B & C \\ 0 & A\end{array}\right)$, where $A$ and $B$ are square matrices with $\operatorname{ind}(A)=r$ and $\operatorname{ind}(B)=s$. Then,

$$
M_{1}^{D}=\left(\begin{array}{cc}
A^{D} & 0 \\
X & B^{D}
\end{array}\right), \quad M_{2}^{D}=\left(\begin{array}{cc}
B^{D} & X \\
0 & A^{D}
\end{array}\right),
$$

where $X=\sum_{i=0}^{r-1}\left(B^{D}\right)^{i+2} C A^{i} A^{\pi}+\sum_{i-0}^{s-1} B^{\pi} B^{i} C\left(A^{D}\right)^{i+2}-B^{D} C A^{D}$.
Lemma 4 (see [24]). Let $M=\left(\begin{array}{ll}A & B \\ C & D\end{array}\right) \in C^{n \times n}$ ( $A$ and $D$ are square matrices). If $S=D-C A^{D} B$ is nonsingular, $A^{\pi} B=0$, and $C A^{\pi}=0$, then

$$
M^{D}=\left(\begin{array}{cc}
A^{D}+A^{D} B S^{-1} C A^{D} & -A^{D} B S^{-1} \\
-S^{-1} C A^{D} & S^{-1}
\end{array}\right) .
$$

\section{The Drazin Inverse of the Sum of Two Matrices}

In this section, we first give the formula for the Drazin inverse of $P+Q$ under some conditions.

Theorem 5. Let $P, Q \in \mathbb{C}^{n \times n}$. If $P^{3} Q=0, P^{2} Q+Q P Q=0$, and $P Q P Q=0$, then

$$
\begin{aligned}
(P+Q)^{D}= & P^{D}+Q^{D}+Q X P+P\left(Q^{D}\right)^{2} \\
& +P^{2}\left(Q^{D}\right)^{3}+P Q\left(P^{D}\right)^{3} \\
& +Q P\left(Q^{D}\right)^{3}+P^{2} Q X P^{D}+P^{2} Q^{D} X P+P Q^{2} X P^{D} \\
& +P Q Q^{D} X P+Q P Q X P^{D}+Q P Q^{D} X P
\end{aligned}
$$

where

$$
\begin{aligned}
X= & \sum_{i=0}^{t-1}\left(Q^{D}\right)^{2 i+4}(P+Q) P^{2 i} P^{\pi} \\
& +\sum_{i=0}^{t-1} Q^{\pi} Q^{2 i}(P+Q)\left(P^{D}\right)^{2 i+4} \\
& -\left(Q^{D}\right)^{2}(P+Q)\left(P^{D}\right)^{2}, \\
t= & \max \left\{\operatorname{ind}\left(P^{2}\right), \operatorname{ind}\left(Q^{2}\right)\right\} .
\end{aligned}
$$

Proof. Using Lemma $1,(A B)^{D}=A\left((B A)^{2}\right)^{D} B$, we have

$$
\begin{aligned}
(P+Q)^{D} & =\left(\left(\begin{array}{ll}
I & Q
\end{array}\right)\left(\begin{array}{l}
P \\
I
\end{array}\right)\right)^{D}=\left(\begin{array}{ll}
I & Q
\end{array}\right)\left(\left(\begin{array}{cc}
P & P Q \\
I & Q
\end{array}\right)^{2}\right)^{D}\left(\begin{array}{l}
P \\
I
\end{array}\right) \\
& =\left(\begin{array}{ll}
I & Q
\end{array}\right)\left(\begin{array}{cc}
P^{2}+P Q & P^{2} Q+P Q^{2} \\
P+Q & Q^{2}+P Q
\end{array}\right)^{D}\left(\begin{array}{l}
P \\
I
\end{array}\right) .
\end{aligned}
$$

Let

$$
M=\left(\begin{array}{cc}
P^{2}+P Q & P^{2} Q+P Q^{2} \\
P+Q & Q^{2}+P Q
\end{array}\right)=E+F,
$$

where

$$
E=\left(\begin{array}{cc}
P^{2} & 0 \\
P+Q & Q^{2}
\end{array}\right), \quad F=\left(\begin{array}{cc}
P Q & P^{2} Q+P Q^{2} \\
0 & P Q
\end{array}\right) .
$$


From $P^{3} Q=0, P^{2} Q+Q P Q=0$ and $P Q P Q=0$, we get $E F=0$, and $F^{2}=0$. Then, applying Lemma 2, we obtain

$$
M^{D}=E^{D}+F\left(E^{D}\right)^{2}
$$

By applying Lemma 3, we have

$$
E^{D}=\left(\begin{array}{cc}
\left(P^{D}\right)^{2} & 0 \\
X & \left(Q^{D}\right)^{2}
\end{array}\right)
$$

where

$$
\begin{aligned}
X= & \sum_{i=0}^{t-1}\left(Q^{D}\right)^{2 i+4}(P+Q) P^{2 i} P^{\pi} \\
& +\sum_{i=0}^{t-1} Q^{\pi} Q^{2 i}(P+Q)\left(P^{D}\right)^{2 i+4} \\
& -\left(Q^{D}\right)^{2}(P+Q)\left(P^{D}\right)^{2}, \\
t= & \max \left\{\operatorname{ind}\left(P^{2}\right), \text { ind }\left(Q^{2}\right)\right\} .
\end{aligned}
$$

Substituting (12) into (11), we get

$$
\begin{aligned}
& M^{D} \\
& =\left(\begin{array}{cc}
\left(P^{D}\right)^{2}+P Q\left(P^{D}\right)^{4}+P^{2} Q X\left(P^{D}\right)^{2} \\
+P^{2} Q^{D} X+P Q^{2} X\left(P^{D}\right)^{2}+P Q Q^{D} X & P^{2}\left(Q^{D}\right)^{3}+P\left(Q^{D}\right)^{2} \\
X+P Q X\left(P^{D}\right)^{2}+P Q^{D} X & \left(Q^{D}\right)^{2}+P\left(Q^{D}\right)^{3}
\end{array}\right) .
\end{aligned}
$$

Substituting (14) into (8), we obtain the result.

Similarly, we give a symmetrical form of Theorem 5 .

Theorem 6. Let $P, Q \in \mathbb{C}^{n \times n}$. If $P Q^{3}=0, P Q^{2}+P Q P=0$, and $P Q P Q=0$, then

$$
\begin{aligned}
(P+Q)^{D}= & P^{D}+Q^{D}+Q X P+\left(P^{D}\right)^{2} Q \\
& +\left(P^{D}\right)^{3} Q^{2}+\left(P^{D}\right)^{3} Q P \\
& +\left(Q^{D}\right)^{3} P Q+Q^{D} X P Q^{2} \\
& +Q^{D} X P^{2} Q+Q X P^{D} Q^{2} \\
& +Q X P^{D} P Q+Q^{D} X P Q P+Q X P^{D} Q P,
\end{aligned}
$$

where

$$
\begin{aligned}
X= & \sum_{i=0}^{t-1}\left(Q^{D}\right)^{2 i+4}(P+Q) P^{2 i} P^{\pi} \\
& +\sum_{i=0}^{t-1} Q^{\pi} Q^{2 i}(P+Q)\left(P^{D}\right)^{2 i+4} \\
& -\left(Q^{D}\right)^{2}(P+Q)\left(P^{D}\right)^{2}, \\
t= & \max \left\{\operatorname{ind}\left(P^{2}\right), \operatorname{ind}\left(Q^{2}\right)\right\} .
\end{aligned}
$$

Next, we present a numerical example to illustrate Theorem 5. This numerical example describes neither the matrices $P$ and $Q$ which do not satisfy the conditions of [10, Theorem 2.2] nor the conditions of [11, Theorem 2.1], but they satisfy the conditions of Theorem 5 . Therefore, we can apply the formula given in Theorem 5 to obtain the Drazin inverse of $P+Q$.

Numerical Example. Consider the matrices $P, Q \in \mathbb{C}^{4 \times 4}$, where

$$
P=\left(\begin{array}{llll}
1 & 0 & 0 & 0 \\
0 & 0 & 0 & 1 \\
0 & 0 & 0 & 0 \\
0 & 0 & 0 & 0
\end{array}\right), \quad Q=\left(\begin{array}{llll}
0 & 0 & 0 & 0 \\
0 & 0 & 1 & 0 \\
0 & 0 & 1 & 0 \\
1 & 0 & 0 & 0
\end{array}\right)
$$

Since $P^{2} Q=0, Q^{2} \neq 0$ and $P Q^{2}=0, P Q P \neq 0$, we know that the conditions of Theorem 2.2 in [10] and Theorem 2.1 in [11] do not hold, respectively. But it satisfies $P^{3} Q=0$, $P^{2} Q+Q P Q=0$, and $P Q P Q=0$. Also, we have

$$
\text { ind }\left(P^{2}\right)=1, \quad \text { ind }\left(Q^{2}\right)=1 \text {, }
$$

$$
P^{D}=\left(\begin{array}{cccc}
1 & 0 & 0 & 0 \\
0 & 0 & 0 & 0 \\
0 & 0 & 0 & 0 \\
0 & 0 & 0 & 0
\end{array}\right), \quad Q^{D}=\left(\begin{array}{llll}
0 & 0 & 0 & 0 \\
0 & 0 & 1 & 0 \\
0 & 0 & 1 & 0 \\
0 & 0 & 0 & 0
\end{array}\right),
$$

$$
X=\left(\begin{array}{llll}
1 & 0 & 0 & 0 \\
0 & 0 & 1 & 0 \\
0 & 0 & 1 & 0 \\
1 & 0 & 0 & 0
\end{array}\right)
$$

So, applying Theorem 5, we get

$$
\begin{aligned}
(P+Q)^{D} & =P^{D}+Q^{D}+P Q\left(P^{D}\right)^{3}+Q X P \\
& =\left(\begin{array}{llll}
1 & 0 & 0 & 0 \\
1 & 0 & 1 & 0 \\
0 & 0 & 1 & 0 \\
1 & 0 & 0 & 0
\end{array}\right)
\end{aligned}
$$

Remark 7. The above example shows that the conditions given in Theorem 5 are satisfied, but the conditions given in $[10,11]$ are not satisfied. 


\section{Drazin Inverse of Some Block Matrices}

In this section, we apply our formula to give the representations for the Drazin inverse of block matrix $M=\left(\begin{array}{ll}A & B \\ C & D\end{array}\right)(A$ and $D$ are square matrices). First, we give the expression of $M^{D}$ under the conditions $A B C=0, B D C=0, D^{2} C=0$, and $C B C=0$, which generalizes the results in $[5,20]$.

Theorem 8. Let $M=\left(\begin{array}{ll}A & B \\ C & D\end{array}\right)$ ( $A$ and $D$ are square matrices), such that $\operatorname{ind}(A)=r$ and $\operatorname{ind}(D)=s$. If $A B C=0, B D C=0$, $D^{2} C=0$, and $C B C=0$, then

$$
M^{D}=P^{D}+\left(\begin{array}{ll}
0 & 0 \\
C & 0
\end{array}\right)\left(P^{D}\right)^{2}+\left(\begin{array}{cc}
B C & 0 \\
D C & 0
\end{array}\right)\left(P^{D}\right)^{3}
$$

where

$$
\begin{gathered}
\left(P^{D}\right)^{i}=\left(\begin{array}{c}
\left(A^{D}\right)^{i} \sum_{j=0}^{i-1}\left(A^{D}\right)^{j} X\left(D^{D}\right)^{i-j-1} \\
0
\end{array}\right), \quad \text { for } i \geq 1 \\
\left.X=\sum_{i=0}^{s-1}\left(D^{D}\right)^{i}\right)^{i+2} B D^{i} D^{\pi} \\
+\sum_{i=0}^{r-1} A^{\pi} A^{i} B\left(D^{D}\right)^{i+2}-A^{D} B D^{D}
\end{gathered}
$$

Proof. Let

$$
M=\left(\begin{array}{ll}
A & B \\
C & D
\end{array}\right)=P+Q
$$

where

$$
P=\left(\begin{array}{cc}
A & B \\
0 & D
\end{array}\right), \quad Q=\left(\begin{array}{ll}
0 & 0 \\
C & 0
\end{array}\right)
$$

From $A B C=0, B D C=0, D^{2} C=0$, and $C B C=0$, we have $P^{3} Q=0, P^{2} Q+Q P Q=0$, and $P Q P Q=0$. We can see that $Q$ is 2-nilpotent, so we get $Q^{D}=0$ and $Q^{\pi}=I$. Applying Theorem 5, we have

$$
M^{D}=P^{D}+Q\left(P^{D}\right)^{2}+P Q\left(P^{D}\right)^{3} .
$$

Using Lemma 3, we obtain the following result:

$$
\left(P^{D}\right)^{i}=\left(\begin{array}{cc}
\left(A^{D}\right)^{i} & \sum_{j=0}^{i-1}\left(A^{D}\right)^{j} X\left(D^{D}\right)^{i-j-1} \\
0 & \left(D^{D}\right)^{i}
\end{array}\right), \quad \text { for } i \geq 1
$$

where $X=\sum_{i=0}^{s-1}\left(A^{D}\right)^{i+2} B D^{i} D^{\pi}+\sum_{i=0}^{r-1} A^{\pi} A^{i} B\left(D^{D}\right)^{i+2}-$ $A^{D} B D^{D}$.

Substituting (25) into (24), we get the result.

Similarly, we consider another splitting of the block matrix $M$ and state another theorem.
Theorem 9. Let $M=\left(\begin{array}{ll}A & B \\ C & D\end{array}\right)$ ( $A$ and $D$ are square matrices), such that $\operatorname{ind}(A)=r$ and $\operatorname{ind}(D)=s$. If $D C B=0, B C B=0$, $A^{2} B=0$, and $C A B=0$, then

$$
M^{D}=P^{D}+\left(\begin{array}{ll}
0 & B \\
0 & 0
\end{array}\right)\left(P^{D}\right)^{2}+\left(\begin{array}{cc}
0 & A B \\
0 & C B
\end{array}\right)\left(P^{D}\right)^{3},
$$

where

$$
\begin{gathered}
\left(P^{D}\right)^{i}=\left(\begin{array}{cc}
\left(A^{D}\right)^{i} & 0 \\
\sum_{j=0}^{i-1}\left(D^{D}\right)^{j} X\left(A^{D}\right)^{i-j-1} & \left(D^{D}\right)^{i}
\end{array}\right), \quad \text { for } i \geq 1 \\
X=\sum_{i=0}^{r-1}\left(D^{D}\right)^{i+2} C A^{i} A^{\pi} \\
+\sum_{i=0}^{s-1} D^{\pi} D^{i} C\left(A^{D}\right)^{i+2}-D^{D} C A^{D}
\end{gathered}
$$

Proof. Let

$$
M=\left(\begin{array}{ll}
A & B \\
C & D
\end{array}\right)=P+Q
$$

where

$$
P=\left(\begin{array}{cc}
A & 0 \\
C & D
\end{array}\right), \quad Q=\left(\begin{array}{cc}
0 & B \\
0 & 0
\end{array}\right)
$$

The remaining proof follows directly from Theorem 8 .

Now, we give the representation for $M^{D}$ when the generalized Schur complement is nonsingular, which generalizes the result in [5].

Theorem 10. Let $M=\left(\begin{array}{cc}A & B \\ C & D\end{array}\right)$ ( $A$ and $D$ are square matrices). If $S=D-C A^{D} B$ is nonsingular, $B C A^{\pi} B=0, D C A^{\pi} B=0$, $A^{2} A^{\pi} B=0$, and $C A A^{\pi} B=0$, then

$$
M^{D}=P^{D}+\left(\begin{array}{cc}
0 & A^{\pi} B \\
0 & 0
\end{array}\right)\left(P^{D}\right)^{2}+\left(\begin{array}{cc}
0 & A A^{\pi} B \\
0 & C A^{\pi} B
\end{array}\right)\left(P^{D}\right)^{3},
$$

where

$$
\begin{gathered}
\left(P^{D}\right)^{j}=\left(P_{1}^{D}\right)^{j}+\sum_{i=1}^{k}\left(P_{1}^{D}\right)^{i+j}\left(\begin{array}{cc}
A^{i} A^{\pi} & 0 \\
C A^{i-1} A^{\pi} & 0
\end{array}\right), \\
P_{1}^{D}=\left(\begin{array}{cc}
A^{D}+A^{D} B S^{-1} C A^{D} & -A^{D} B S^{-1} \\
-S^{-1} C A^{D} & S^{-1}
\end{array}\right), \quad k=\operatorname{ind}(A) .
\end{gathered}
$$

Proof. Let

$$
M=\left(\begin{array}{ll}
A & B \\
C & D
\end{array}\right)=P+Q,
$$


where

$$
P=\left(\begin{array}{cc}
A & A A^{D} B \\
C & D
\end{array}\right), \quad Q=\left(\begin{array}{cc}
0 & A^{\pi} B \\
0 & 0
\end{array}\right)
$$

From $B C A^{\pi} B=0, D C A^{\pi} B=0, A^{2} A^{\pi} B=0$, and $C A A^{\pi} B=0$, it is obvious that $P^{3} \mathrm{Q}=0, P^{2} \mathrm{Q}+\mathrm{QPQ}=0$, and $P Q P Q=0$. We can see that $Q$ is 2-nilpotent, so we get $Q^{D}=0$ and $Q^{\pi}=I$. Applying Theorem 5, we have

$$
M^{D}=P^{D}+Q\left(P^{D}\right)^{2}+P Q\left(P^{D}\right)^{3} .
$$

Let $P=P_{1}+P_{2}$, where

$$
P_{1}=\left(\begin{array}{cc}
A^{2} A^{D} & A A^{D} B \\
C A A^{D} & D
\end{array}\right), \quad P_{2}=\left(\begin{array}{ll}
A A^{\pi} & 0 \\
C A^{\pi} & 0
\end{array}\right) .
$$

Obviously, $P_{2} P_{1}=0$ and $P_{2}^{k+1}=0$, where $k=\operatorname{ind}(A)$. By Lemma 2, we have

$$
\begin{array}{r}
\left(P^{D}\right)^{j}=\left(P_{1}^{D}\right)^{j}+\sum_{i=1}^{k}\left(P_{1}^{D}\right)^{i+j} P_{2}^{i}, \quad P_{2}^{i}=\left(\begin{array}{cc}
A^{i} A^{\pi} & 0 \\
C A^{i-1} A^{\pi} & 0
\end{array}\right), \\
i \geq 1 .
\end{array}
$$

For $P_{1}$, we get that $S=D-C A A^{D}\left(A^{2} A^{D}\right)^{D} A A^{D} B=D-C A^{D} B$ is nonsingular, $\left(A^{2} A^{D}\right)^{\pi} A A^{D} B=0$, and $C A A^{D}\left(A^{2} A^{D}\right)^{\pi}=0$. Using Lemma 4 , we obtain the following result:

$$
P_{1}^{D}=\left(\begin{array}{cc}
A^{D}+A^{D} B S^{-1} C A^{D} & -A^{D} B S^{-1} \\
-S^{-1} C A^{D} & S^{-1}
\end{array}\right) .
$$

From the above equation, we obtain the result in Theorem 10.

In the same way, we consider another splitting of the block matrix $M$ and present next theorem.

Theorem 11. Let $M=\left(\begin{array}{ll}A & B \\ C & D\end{array}\right)$ ( $A$ and $D$ are square matrices). If $S=D-C A^{D} B$ is nonsingular, $A B C A^{\pi}=0, B D C A^{\pi}=0$, $D^{2} C A^{\pi}=0$, and $A^{\pi} B C A^{\pi}=0$, then

$$
M^{D}=P^{D}+\left(\begin{array}{cc}
0 & 0 \\
C A^{\pi} & 0
\end{array}\right)\left(P^{D}\right)^{2}+\left(\begin{array}{cc}
B C A^{\pi} & 0 \\
D C A^{\pi} & 0
\end{array}\right)\left(P^{D}\right)^{3},
$$

where

$$
\begin{gathered}
\left(P^{D}\right)^{j}=\left(P_{1}^{D}\right)^{j}+\sum_{i=1}^{k}\left(\begin{array}{cc}
A^{i} A^{\pi} & A^{i-1} A^{\pi} B \\
0 & 0
\end{array}\right)\left(P_{1}^{D}\right)^{i+j}, \\
P_{1}^{D}=\left(\begin{array}{cc}
A^{D}+A^{D} B S^{-1} C A^{D} & -A^{D} B S^{-1} \\
-S^{-1} C A^{D} & S^{-1}
\end{array}\right), \quad k=\operatorname{ind}(A) .
\end{gathered}
$$

Proof. Let

$$
M=\left(\begin{array}{ll}
A & B \\
C & D
\end{array}\right)=P+Q,
$$

where

$$
P=\left(\begin{array}{cc}
A & B \\
C A A^{D} & D
\end{array}\right), \quad Q=\left(\begin{array}{cc}
0 & 0 \\
C A^{\pi} & 0
\end{array}\right)
$$

The remaining proof is similar to that of Theorem 10.

\section{Numerical Examples}

In this section, two numerical examples are given to illustrate Theorems 8 and 10.

Example 1. Consider the block matrix $M=\left(\begin{array}{ll}A & B \\ C & D\end{array}\right) \in \mathbb{C}^{4 \times 4}$, where

$$
\begin{array}{ll}
A=\left(\begin{array}{ll}
1 & 0 \\
0 & 0
\end{array}\right), & B=\left(\begin{array}{ll}
0 & 0 \\
0 & 1
\end{array}\right), \\
C=\left(\begin{array}{ll}
0 & 0 \\
1 & 0
\end{array}\right), & D=\left(\begin{array}{ll}
1 & 0 \\
1 & 0
\end{array}\right) .
\end{array}
$$

Since $B C \neq 0$ and $B C A \neq 0$, the representation for $M^{D}$ fail to apply in $[9,11,20-22]$, respectively. But it satisfies $A B C=0$, $B D C=0, D^{2} C=0$, and $C B C=0$. Also, we have

$$
\begin{aligned}
A^{D}=\left(\begin{array}{ll}
1 & 0 \\
0 & 0
\end{array}\right), & A^{\pi}=\left(\begin{array}{ll}
0 & 0 \\
0 & 1
\end{array}\right), \\
D^{D}=\left(\begin{array}{ll}
1 & 0 \\
1 & 0
\end{array}\right), & D^{\pi}=\left(\begin{array}{cc}
0 & 0 \\
-1 & 1
\end{array}\right) .
\end{aligned}
$$

Then, applying Theorem 8 , we obtain

$$
M^{D}=\left(\begin{array}{llll}
1 & 0 & 0 & 0 \\
1 & 0 & 1 & 0 \\
0 & 0 & 1 & 0 \\
1 & 0 & 1 & 0
\end{array}\right)
$$

Remark 12. The above example shows that the conditions given in Theorem 8 are satisfied, but the conditions given in $[9,11,20-22]$ are not satisfied.

Example 2. Consider the block matrix $M=\left(\begin{array}{cc}A & B \\ C & D\end{array}\right) \in \mathbb{C}^{5 \times 5}$, where

$$
\begin{aligned}
A=\left(\begin{array}{ll}
1 & 1 \\
0 & 0
\end{array}\right), & B=\left(\begin{array}{lll}
1 & 2 & 1 \\
0 & 0 & 0
\end{array}\right), \\
C=\left(\begin{array}{ll}
1 & 2 \\
1 & 0 \\
0 & 1
\end{array}\right), & D=\left(\begin{array}{lll}
2 & 2 & 1 \\
1 & 3 & 1 \\
0 & 0 & 1
\end{array}\right) .
\end{aligned}
$$


By computing, we get that $S=D-C A^{D} B$ is nonsingular and $B C A^{\pi} B=0, D C A^{\pi} B=0, A^{2} A^{\pi} B=0$, and $C A A^{\pi} B=0$. Also, we have

$$
\begin{aligned}
& \text { ind }(A)=1, \quad A^{D}=\left(\begin{array}{ll}
1 & 1 \\
0 & 0
\end{array}\right) \text {, } \\
& A^{\pi}=\left(\begin{array}{cc}
0 & -1 \\
0 & 1
\end{array}\right), \quad S^{-1}=\left(\begin{array}{lll}
1 & 0 & 0 \\
0 & 1 & 0 \\
0 & 0 & 1
\end{array}\right) \text {, } \\
& P_{1}^{D}=\left(\begin{array}{ccccc}
4 & 4 & -1 & -2 & -1 \\
0 & 0 & 0 & 0 & 0 \\
-1 & -1 & 1 & 0 & 0 \\
-1 & -1 & 0 & 1 & 0 \\
0 & 0 & 0 & 0 & 1
\end{array}\right) \\
& P^{D}=\left(\begin{array}{ccccc}
4 & 4 & -1 & -2 & -1 \\
0 & 0 & 0 & 0 & 0 \\
-1 & 0 & 1 & 0 & 0 \\
-1 & -2 & 0 & 1 & 0 \\
0 & 1 & 0 & 0 & 1
\end{array}\right)
\end{aligned}
$$

Then, applying Theorem 10, we get

$$
M^{D}=\left(\begin{array}{ccccc}
4 & 4 & -1 & -2 & -1 \\
0 & 0 & 0 & 0 & 0 \\
-1 & 0 & 1 & 0 & 0 \\
-1 & -2 & 0 & 1 & 0 \\
0 & 1 & 0 & 0 & 1
\end{array}\right)
$$

\section{Conflict of Interests}

The authors declare that there is no conflict of interests regarding the publication of this paper.

\section{Acknowledgments}

The authors thank the reviewers for the valuable comments. This work was supported by the Ph.D. Programs Foundation of Ministry of Education of China (Grant no. 20110191110033).

\section{References}

[1] A. Ben-Israel and T. N. E. Greville, Generalized Inverses: Theory and Applications, Springer, New York, NY, USA, 2nd edition, 2003.

[2] S. L. Campbell and C. D. Meyer Jr., Generalized Inverses of Linear Transformations, Dover, New York, NY, USA, 1991.

[3] X. Chen and R. E. Hartwig, "The group inverse of a triangular matrix," Linear Algebra and Its Applications, vol. 237/238, pp. 97108, 1996.

[4] Y. Wei, X. Li, and F. Bu, "A perturbation bound of the Drazin inverse of a matrix by separation of simple invariant subspaces," SIAM Journal on Matrix Analysis and Applications, vol. 27, no. 1, pp. 72-81, 2005.

[5] R. Hartwig, X. Li, and Y. Wei, "Representations for the Drazin inverse of a $2 \times 2$ block matrix," SIAM Journal on Matrix Analysis and Applications, vol. 27, no. 3, pp. 757-771, 2005.
[6] M. P. Drazin, "Pseudo-inverses in associative rings and semigroups," The American Mathematical Monthly, vol. 65, pp. 506514, 1958.

[7] R. E. Hartwig, G. Wang, and Y. Wei, "Some additive results on Drazin inverse," Linear Algebra and Its Applications, vol. 322, no. 1-3, pp. 207-217, 2001.

[8] N. Castro González, "Additive perturbation results for the Drazin inverse," Linear Algebra and Its Applications, vol. 397, pp. 279-297, 2005.

[9] N. Castro-González, E. Dopazo, and M. F. Martínez-Serrano, "On the Drazin inverse of the sum of two operators and its application to operator matrices," Journal of Mathematical Analysis and Applications, vol. 350, no. 1, pp. 207-215, 2009.

[10] M. F. Martínez-Serrano and N. Castro-González, "On the Drazin inverse of block matrices and generalized Schur complement," Applied Mathematics and Computation, vol. 215, no. 7, pp. 2733-2740, 2009.

[11] H. Yang and X. Liu, "The Drazin inverse of the sum of two matrices and its applications," Journal of Computational and Applied Mathematics, vol. 235, no. 5, pp. 1412-1417, 2011.

[12] C. Bu, C. Feng, and S. Bai, "Representations for the Drazin inverses of the sum of two matrices and some block matrices," Applied Mathematics and Computation, vol. 218, no. 20, pp. 10226-10237, 2012.

[13] N. Castro-González, J. Robles, and J. Y. Vélez-Cerrada, “Characterizations of a class of matrices and perturbation of the Drazin inverse," SIAM Journal on Matrix Analysis and Applications, vol. 30, no. 2, pp. 882-897, 2008.

[14] N. Castro-González and J. Y. Vélez-Cerrada, "On the perturbation of the group generalized inverse for a class of bounded operators in Banach spaces," Journal of Mathematical Analysis and Applications, vol. 341, no. 2, pp. 1213-1223, 2008.

[15] Q. Xu, C. Song, and Y. Wei, "The stable perturbation of the Drazin inverse of the square matrices," SIAM Journal on Matrix Analysis and Applications, vol. 31, no. 3, pp. 1507-1520, 2009.

[16] C. Y. Deng, "The Drazin inverses of sum and difference of idempotents," Linear Algebra and Its Applications, vol. 430, no. 4, pp. 1282-1291, 2009.

[17] X. Liu, L. Xu, and Y. Yu, "The representations of the Drazin inverse of differences of two matrices," Applied Mathematics and Computation, vol. 216, no. 12, pp. 3652-3661, 2010.

[18] C. Deng, D. S. Cvetković-Ilić, and Y. Wei, "Some results on the generalized Drazin inverse of operator matrices," Linear and Multilinear Algebra, vol. 58, no. 3-4, pp. 503-521, 2010.

[19] A. Shakoor, H. Yang, and I. Ali, "The Drazin inverses of the sum of two matrices and block matrix," Journal of Applied Mathematics \& Informatics, vol. 31, no. 3-4, pp. 343-352, 2013.

[20] D. S. Djordjević and P. S. Stanimirović, "On the generalized Drazin inverse and generalized resolvent," Czechoslovak Mathematical Journal, vol. 51, no. 3, pp. 617-634, 2001.

[21] D. S. Cvetković-Ilić, "A note on the representation for the Drazin inverse of $2 \times 2$ block matrices," Linear Algebra and Its Applications, vol. 429, no. 1, pp. 242-248, 2008.

[22] J. Ljubisavljević and D. S. Cvetković-Ilić, "Additive results for the Drazin inverse of block matrices and applications," Journal of Computational and Applied Mathematics, vol. 235, no. 12, pp. 3683-3690, 2011.

[23] J. M. Miao, "Some results on the Drazin inverses of partitioned matrices," Journal of Shanghai Teachers University, vol. 18, no. 2, pp. 25-31, 1989. 
[24] Y. Wei, "Expressions for the Drazin inverse of a $2 \times 2$ block matrix," Linear and Multilinear Algebra, vol. 45, no. 2-3, pp. 131146, 1998.

[25] C. Y. Deng, "Generalized Drazin inverses of anti-triangular block matrices," Journal of Mathematical Analysis and Applications, vol. 368, no. 1, pp. 1-8, 2010.

[26] C. D. Meyer, Jr. and N. J. Rose, "The index and the Drazin inverse of block triangular matrices," SIAM Journal on Applied Mathematics, vol. 33, no. 1, pp. 1-7, 1977. 


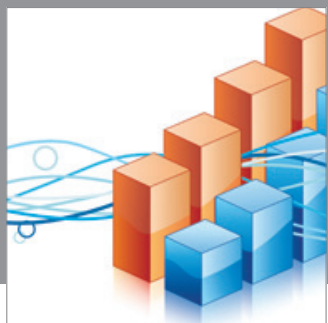

Advances in

Operations Research

mansans

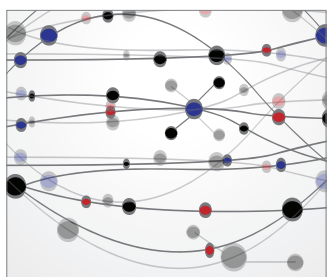

The Scientific World Journal
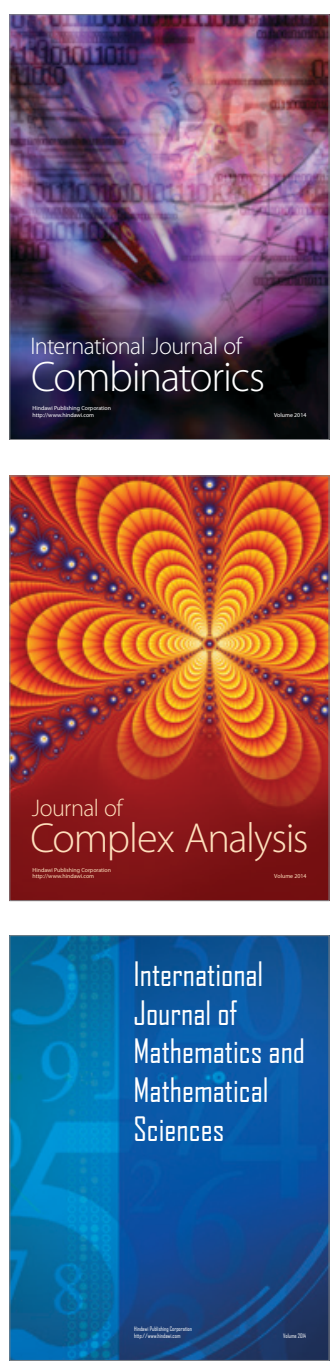
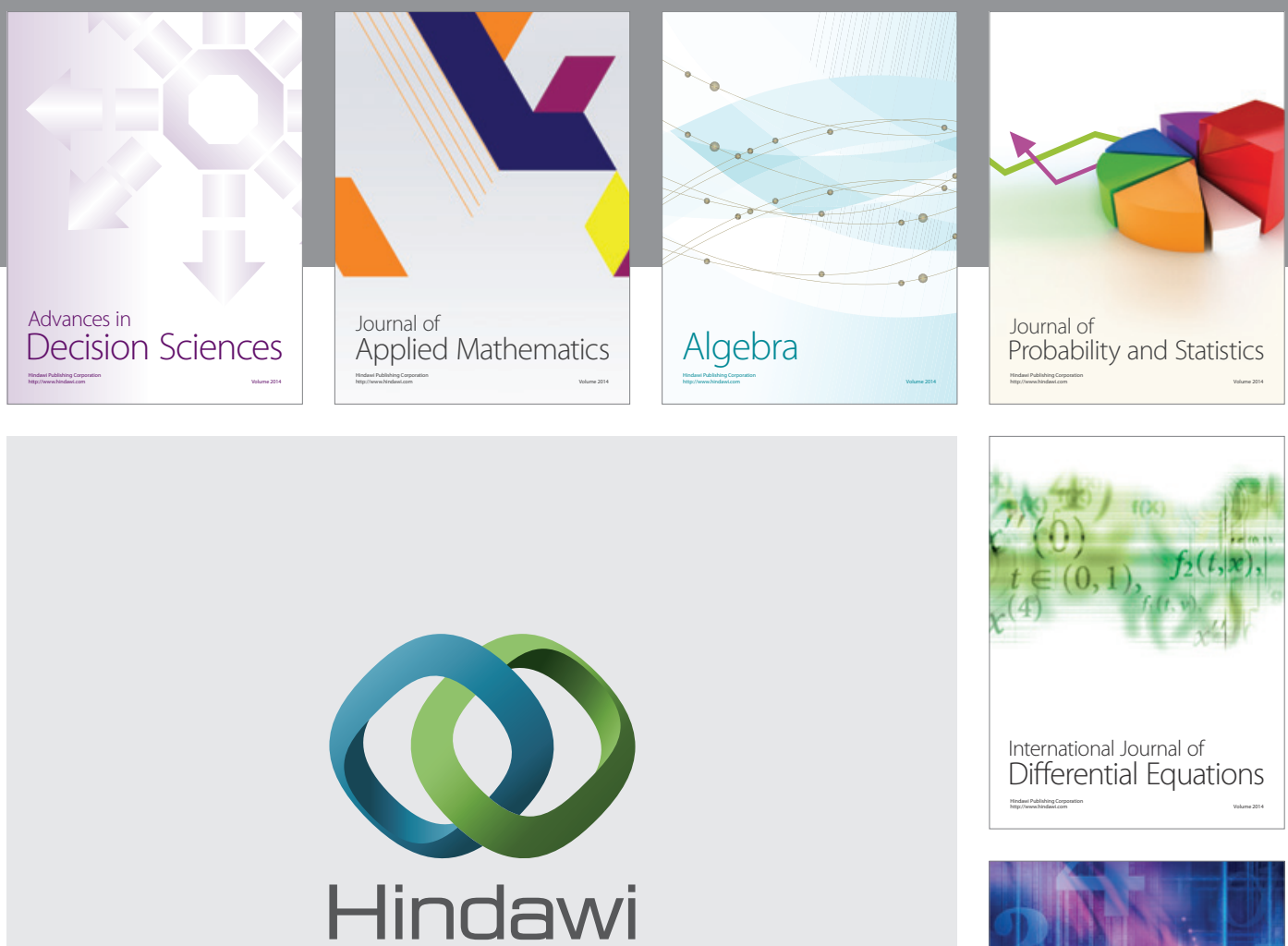

Submit your manuscripts at http://www.hindawi.com
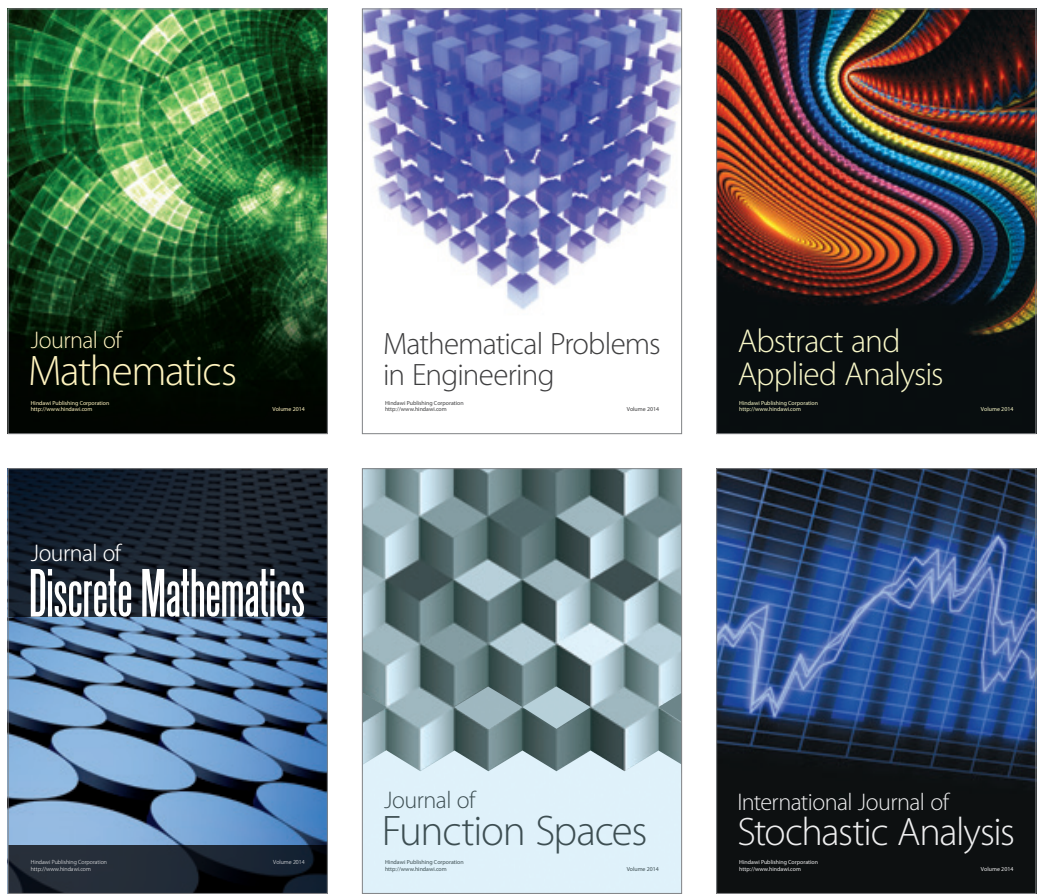

Journal of

Function Spaces

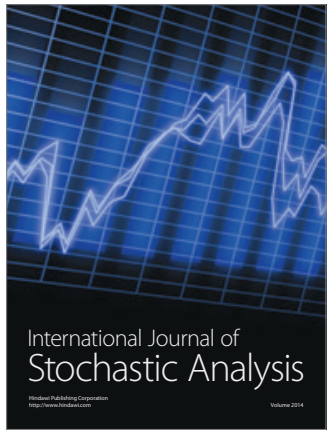

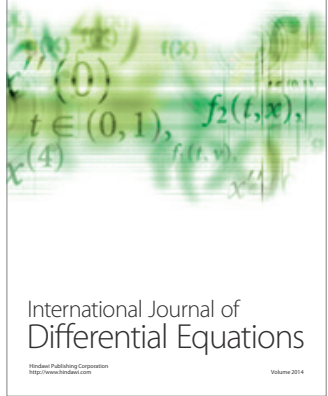
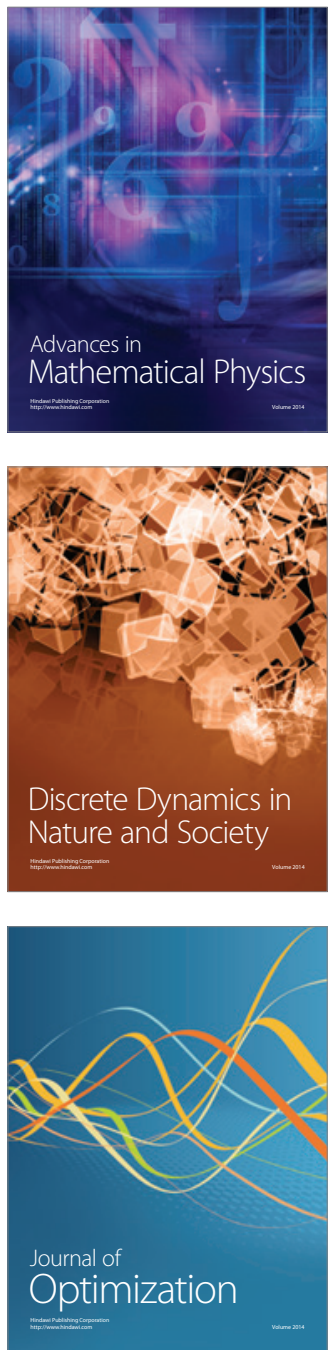